\title{
"CUIDADO COM QUEM VOCÊ FALA NA INTERNET": MEDIAÇÃO PARENTAL PELO OLHAR DE PRÉ-ADOLESCENTES
}

\author{
'BE CAREFUL TO WHOM YOU SPEAK ONLINE': \\ PARENTAL MEDIATION THROUGH \\ PRE-ADOLESCENTS' EYES
}

Teresa Sofia Castro ${ }^{1}$

\begin{abstract}
RESUMO: Quando falamos de telas com ligação à Internet, que as crianças usam para lazer e comunicação com seus pares, e da mediação parental, a literatura identifica que, nesse assunto, as crianças ainda são pouco ouvidas. Este artigo resulta de uma tese de doutorado na qual ouvimos as perspectivas críticas de pré-adolescentes portugueses, vindas de famílias de rendas média e média-baixa. Procuramos analisar dois tópicos centrais: o papel influente da mídia nas percepções dos pais e como os pré-adolescentes respondem à mediação parental.
\end{abstract}

Palavras-chave: Mediação parental. Crianças. Pré-adolescentes. Comunicação conectada. Internet.

\begin{abstract}
When talking about devices with Internet connection, which children use for leisure and communication with their peers, and parental mediation, the literature identifies that, on this subject, children are still scarcely heard. This paper is the result of a doctoral dissertation where we listened to the critical perspectives of Portuguese pre-adolescents from middle and lower-middle income families. We aimed at analyzing two central topics: the influential role of the media in parents' perceptions, and how pre-adolescents respond to parental mediation.
\end{abstract}

Keywords: Parental mediation. Children. Pre-adolescents. Connected communication. Internet.

\footnotetext{
1.Universidade Nova de Lisboa - Lisboa, Portugal.

*Autora correspondente: teresa.sofia.castro@gmail.com

Estudo financiado pela Fundação Portuguesa para a Ciência e Tecnologia, no âmbito do QREN-POPH e pelo Ministério da Ciência, Tecnologia e Ensino Superior (ref. SFRH/BD/68288/2010).

Número temático organizado por: Gilka Girardello, Adriana Hoffmann e Inês Vitorino Sampaio
} 


\section{Introdução}

Pouca teoria sobre mediação parental é apoiada nas perspectivas das crianças (HADDON, 2015) e em seu direito a ser ouvidas em assuntos que lhes dizem respeito. A contribuição central deste artigo consiste, primeiramente, em demonstrar como os mais jovens percebem e refletem a mediação parental, partindo de suas vozes. Em segundo lugar, o artigo chama a atenção para o fato de as crianças terem os próprios interesses, expectativas e experiências no consumo digital, pelo que ativam estratégias para contornar a mediação parental. Além disso, mostra-se aqui que as percepções dos pais são, muitas vezes, influenciadas por pânicos sociais disseminados e explorados, graças à sua atratividade, pela mídia e pelos discursos públicos, mas que resultam num desencontro de expectativas.

Este artigo parte de dados qualitativos coletados entre 2013 e 2014 para uma pesquisa de doutorado, os quais mantêm atualidade e pertinência para reflexão. Para chegar às percepções das crianças, foram utilizadas abordagens participativas (CASTRO, 2015) e, partindo de suas experiências cotidianas, as crianças falaram sobre as complexidades contidas no consumo de mídia digital, na mediação parental e na participação digital (CASTRO, 2015). Pretendeu-se examinar esses dados e responder às seguintes questões de pesquisa: "Estarão os discursos da mídia moldando as percepções dos pais sobre o consumo de mídia digital, do ponto de vista das crianças?"; "Como estão os pré-adolescentes respondendo às abordagens de mediação parental mais intrusivas?"

\section{Crianças, Mediação Parental e Mídia Digital}

Embora as crianças não sejam sujeitos centrais em análises sociais altamente influentes, feitas por autores europeus como Beck (1992) ou Giddens (1990), os diálogos teóricos desses autores sobre o que designam como "sociedade de risco" oferecem leituras pertinentes e esclarecedoras, as quais permitem compreender o clima de alarme e ansiedade que parece moldar as relações contemporâneas ocidentais entre adultos e crianças. O risco se tornou uma representação cultural e uma interpretação da vida no início do século XXI, muito encorajadas pela mídia (FUREDI, 1997).

As construções sociais de risco, como Bond (2014) explica, tornaram-se centrais não apenas na compreensão da sociedade, mas se expandiram para explicar a infância e os estilos parentais nas sociedades ocidentais da modernidade tardia. Tal atitude pessimista afeta a relação entre adultos e crianças, incentivando a construção de mais problemas sociais, pela falta de confiança na comunidade (indivíduos, instituições e governos); favorecendo o caminho de suspeita, vulnerabilidade e individualização; e aumentando e legitimando estratégias de supervisão e de policiamento, sob uma ilusão de controle (PIPER et al., 2006; STOKES, 2009; WONG, 2010). A isso Furedi (2001) classificou como "parentalidade paranoica": uma preocupação ocidental obsessiva com a segurança infantil. Quinze anos depois, Lim (2016), à luz de inovações tecnológicas mais recentes, discute como a nuvem (cloud) e a conectividade ininterrupta moldaram os hábitos de consumo e como isso desafia essa vigilância parental obsessiva.

Nessa atmosfera social, em que o risco é considerado uma realidade omnipresente (FRANKLIN; CROMBY, 2009), atividades outrora classificadas como saudáveis, criativas e divertidas são hoje assombradas por uma infinidade de riscos: exposição solar (FUREDI, 1997), falar com desconhecidos (PAIN, 2006) e a pedofilia (FRANKLIN; CROMBY, 2009) são exemplos comuns. Como resultado, esses e outros temores estão expulsando, de novo, as crianças da esfera da vida pública (BOYD; HARGITTAI, 2013; PAIN, 2006), inibindo-as de experimentar o mundo exterior e de desenvolver habilidades sociais, emocionais e de resiliência (GILL, 2007; SUTTERBY, 2009; SUTTON, 2008). 
Agravando o cenário (do ponto de vista dos adultos) estão os dispositivos portáteis e conectados. Primeiramente, à medida que as crianças crescem, elas se tornam digitalmente mais autônomas e seus interesses digitais mudam muito rapidamente. Em segundo lugar, os dispositivos portáteis e sempre conectados dificultam o gerenciamento de interações digitais (DUERAGER; LIVINGSTONE, 2014). Em terceiro, tendências de consumo mais privadas (HADDON, 2013), fluidas e "em movimento" colocam à prova regras que serviram no passado, num cenário baseado na mídia tradicional.

Com os avanços tecnológicos dos últimos anos (Wi-Fi, portabilidade, redes sociais, Internet das Coisas, inteligência artificial, YouTubers e influencers), a paternidade tornou-se um trabalho extenuante, em tempo integral, repleto de dúvidas e expectativas novas e "transcendentes" (LIM, 2016), impossíveis de atender. Os pais se tornaram guardiões de seus filhos desde que acordam até a hora de dormir. Segundo alguns autores, à medida que as crianças foram desencorajadas de correr riscos e excluídas da esfera pública, voltaram-se para a Internet, de modo a recuperar um novo espaço público, no qual podem encontrar-se com seus pares e se divertir juntos (BOYD; HARGITTAI, 2013).

É importante considerar, ainda, o contexto português para entender os dados aqui discutidos: Qual tem sido a abordagem dos pais portugueses quanto às atividades digitais de seus filhos pré-adolescentes? Quais são suas principais preocupações? Os relatórios EU Kids Online mostram (DUERAGER; LIVINGSTONE, 2014; O’NEILL, 2014; VANDONINCK et al. 2013) que os portugueses estão no grupo de pais cujas abordagens de mediação são mais restritivas. Apesar de ter resultados positivos, essa é a abordagem menos eficaz em termos de proteção (HELSPER et al., 2013; O’NEILL, 2014). Sendo o acesso à Internet um direito humano fundamental - reconhecido pela Organização das Nações Unidas -, a falta desse acesso fecha o caminho para um desenvolvimento cognitivo e psicossocial benéfico, disponível no mundo social on-line (TYNES, 2007). Isso pode causar níveis mais altos de dano para os indivíduos quando deparam com o risco sem ter experiência digital e competências para lidar com esses desafios (HELSPER et al., 2013).

As abordagens restritivas também espelham uma construção romântica da infância (JAMES; JENKS, 1996; JAMES, 2010): a criança vulnerável, inocente e sem agência, que precisa de proteção. Essa visão nostálgica legitima intervenções de policiamento e vigilância pelos pais - que ressoam o conceito de governamentalidade de Foucault (1979) -, nas quais os pais exercem relações de poder panópticas sobre os filhos.

Boyd e Hargittai (2013) identificam três tipos principais de riscos, que tendem a dominar o discurso público e influenciar a mediação parental: contato por desconhecidos; intimidação e assédio; e exposição a conteúdo inapropriado (p. ex., pornografia e violência). Corroborando isso, Ponte e Simões (2009), do EU Kids Online Portugal, apontam que dois desses riscos tendem a dominar também as preocupações e as decisões de supervisão dos pais portugueses: contato e conteúdo visualizado.

\section{Metodologia}

Este artigo tem como alicerce as perspectivas das crianças sobre a mediação parental. $\mathrm{O}$ trabalho de campo envolveu 41 crianças, com idades variando entre 10 e 12 anos, vindas de famílias de renda média e média-baixa - um grupo sub-representado na literatura (LIVINGSTONE; BULGER, 2013).

Tal faixa etária corresponde ao estágio da pré-adolescência, quando os indivíduos adquirem maior autonomia cultural (PASQUIER, 2008) e possuem seu próprio dispositivo para uso privado e comunicação pessoal.

A realidade é uma construção social feita pelos atores para darem sentido às suas práticas e contextos de vida. Assim, recorremos, durante este trabalho, ao uso de técnicas dialógicas, flexíveis e reflexivas, que promoveram a participação das crianças na pesquisa e o equilíbrio de poder entre pesquisador 
e crianças (p. ex., jogos, desenho e dramatização, grupos focais, entrevistas individuais e observação participante). Por meio dessas técnicas, as crianças expressaram suas perspectivas sobre assuntos cotidianos, atividades digitais, mediação parental e interações digitais.

Para proteger seus direitos de privacidade e confidencialidade, foram ocultadas identidades e informações pessoais. Suas identidades foram substituídas por pseudônimos.

Para organizar e dar sentido aos dados coletados, utilizamos dois métodos compatíveis com a tradição construtivista (GUBA; LINCOLN, 1998): análise temática (BRAUN; CLARKE, 2006) e análise narrativa (RIESSMAN, 2008). Para este artigo, os dados selecionados foram organizados em dois temas: o papel influente da mídia nas percepções dos pais segundo as crianças e como os pré-adolescentes respondem à mediação parental.

\section{Discussão de Resultados}

\section{O Papel Influente da Mídia nas Percepções dos Pais}

O risco relativo às crianças contatadas por desconhecidos é amplamente divulgado na mídia (LIVINGSTONE et al., 2015) e um dos que mais alimentam as preocupações dos pais. Alice (10 anos) contounos como o pai agiu quando descobriu que ela havia aceitado um pedido de amizade de um desconhecido numa rede social:

Enviaram-me um pedido de amizade. Eu aceitei, mas não conhecia essa pessoa e, um dia, a pessoa enviou-me várias mensagens. Eu não respondi, mas o meu pai viu e disse: "Alice, você está de castigo. Não deveria aceitar solicitações de amizade de pessoas que não conhece.”

(Alice, 10 anos)

No caso de Alice, pelo menos em curto prazo, o policiamento de seu pai pode mostrar-se eficaz: Alice confirma que, desde então, tem obedecido, bem como não aceitaria mais pedidos de amizade de desconhecidos. Se esse exemplo confirma o argumento de Livingstone e Helsper (2008) de que a restrição pode manter as crianças mais seguras, por outro lado, revela que Alice e seu pai poderiam ter transformado esse acontecimento numa oportunidade pedagógica, ajudando a menor a lidar com potenciais situações de risco. Construindo competências digitais e laços de confiança, o pai poderia ajudá-la a avaliar quando e se um estranho pode (ou não) representar (sempre) um risco, tal como acontece nas interações offline. Sua decisão, pelo contrário, apenas reforçou o foco no risco e no problema, em vez da solução.

Ter um perfil numa rede social ou jogar on-line, na verdade, abre espaço para desconhecidos (apesar de idade, sexo ou intenções) entrarem em contato com as crianças (e vice-versa), conforme relatado pelos pré-adolescentes (CASTRO, 2015).

Mais acesso e uso implicam mais contato com situações de risco. Para lidar com os inevitáveis problemas, os pais têm, habitualmente, uma destas reações: proibir ou negociar. Quando optam por negociar, por vezes permitem, por exemplo, que a criança tenha um perfil numa rede social, mas sob a condição de que as solicitações de amizade sejam validadas pelos pais ou irmãos mais velhos, como acontece com Marina (12 anos). Seus pais confiam no filho mais velho para ser o mediador digital, orientando e supervisionando as atividades digitais da irmã mais nova: "Prometi ao meu irmão não aceitar pedidos de desconhecidos." No entanto, e provando a agência das crianças para decidir por si e contornar as restrições dos pais, Teresa (11 anos) mentiu e, com a ajuda de uma prima, criou um perfil sem autorização e conhecimento dos pais. Quase todas as crianças envolvidas nesta pesquisa tinham um perfil digital abaixo da idade mínima requerida (13 anos). 
As crianças compartilharam que, apesar de conhecerem os riscos, muitas vezes aceitam solicitações de pessoas que não conhecem, seja em redes sociais, seja em jogos on-line. Nessa faixa etária, o capital social também é medido pelo número de seguidores e "amigos" digitais. Assim, os entrevistados justificam suas ações não como risco, mas como oportunidades arriscadas, que eles controlam por medidas de segurança planejadas (p. ex., não se envolver em conversas com as pessoas desconhecidas - decisão discutível e ambivalente, já que, ao mesmo tempo, consentem a esses desconhecidos o acesso a diversas informações pessoais que podem ser lidas, baixadas e disseminadas, como publicações, interesses e fotografias).

Eu aceito pedidos de desconhecidos, mas não ... [interrompida]. (Diana, 10 anos)

[Finalizando a frase de Diana] Mas ela nunca fala com eles. (Francisco, 12 anos)

Sobre a mediação parental, duas meninas apresentaram sua percepção crítica e estabeleceram uma relação entre supervisão e cobertura da mídia, confirmando seu poder nesse domínio (ANDERSON, 2006):

Quando meus pais assistem a essas notícias [sobre riscos on-line], eles me dizem para ter cuidado com quem eu falo na Internet (Joana, 10 anos).

Lara (10 anos) acrescenta, em tom teatral:

Minha mãe todos os dias me azucrina com todos esses perigos da Internet. (Lara, 10 anos)

Com a ajuda das crianças, produzimos uma lista com as principais preocupações que os pais expressam junto delas. A lista foi organizada de acordo com o critério de frequência: contato por desconhecidos; pornografia on-line; violência; vírus; dependência de jogos e redes sociais. Essa conclusão é apoiada na literatura por Ponte et al. (2017) e em um relatório EU Kids Online (LIVINGSTONE et al., 2012), em que os pais portugueses demonstram ter o dobro de preocupação, quando comparados com outros pais europeus, sobre dois tipos de riscos: contato com desconhecidos e conteúdos sexuais.

Se as preocupações dos pais permanecem estáveis ao longo dos anos, as preocupações das crianças estão mais atualizadas e sofisticadas. Elas se preocupam com: intimidação cibernética; acesso de desconhecidos a suas informações pessoais; rastreamento por software de localização; roubo de senhas; acesso de hackers pela webcam; contato de desconhecidos; pornografia; sequestro (quando se encontram com contatos on-line); roubo de identidade; perfis fake; e chantagem causada por fotos enviadas (p. ex., em casos de sexting). Esses são exemplos que provam como as preocupações das crianças são mais específicas e complexas, refletindo os avanços tecnológicos e a incorporação da cultura digital em suas vidas e interações diárias.

\section{Como os Pré-adolescentes Respondem à Mediação Parental}

À medida que a tecnologia cria mais oportunidades para que as crianças escapem à supervisão parental (navegação em modo incógnito; mobilidade; dispositivos menores; aplicativos que os pais não conhecem ou não sabem usar etc.), mais alguns pais sentem sua supervisão ameaçada. Outros, por sua vez, tiram proveito da arquitetura das plataformas para supervisionar e controlar os passos digitais da criança. O relato a seguir ilustra os tipos de tensão intergeracional que podem surgir quando o monitoramento incentiva os filhos a agir para proteger suas privacidade e participação digital: 
Eu sou amiga deles [pais]. Mas às vezes não publico coisas por causa deles. E você sabe por quê?

Olhe essa situação: Rosa me envia um pedido de amizade e eu aceito. Ela se torna minha amiga, certo? Minha mãe vê e então me pergunta: "Você conhece essa pessoa?” E eu respondo: “Sim, mãe." Então ela diz: “Mas eu não." Olha, ela é realmente irritante. Quer saber tudo. Estamos bem melhor sem pais bisbilhotando (Eva, 12 anos).

O modo como Eva lida com a vigilância parental ressoa a individualização ocidental, reivindicada por Beck (1992), a reflexividade discutida por Giddens (1990) e a necessidade de permanecer no controle da situação, conforme argumentado por Boyd (2014). A combinação desses três aspectos é significativa para entender como Eva examina e reflete suas práticas digitais para evitar conflitos resultantes da estrutura panóptica da própria rede social, além de revelar falta de confiança (nesse caso, bidirecional) entre mãe e filha - um corolário da sociedade da modernidade tardia -, resultado de como Eva espera que a mãe se comporte.

Outras crianças na pesquisa relataram um papel ativo na maneira como respondem às práticas de mediação dos pais, recorrendo a estratégias de reatância mais ou menos criativas para mitigar esse diferencial de poder e contornar esquemas de controle (CASTRO, 2015). A mediação parental pode ser mais aberta ou encoberta, mas as descobertas empíricas demonstram que as crianças notam quando e como seus pais as vigiam. Assim, quando os pais usam esquemas que restringem sua liberdade on-line, a privacidade, a participação e os direitos de autoexpressão, as crianças procuram ativamente escapar a esse controle.

Para a geração nascida no novo milênio, o valor desses dispositivos sempre conectados vai além de seu uso ou de sua utilidade: eles reforçam sentimentos tribais e de pertencimento (MAFFESOLI, 1996). Quando privados de liberdade digital, esses indivíduos optam por migrar para plataformas menos familiares para os adultos. Como nômades digitais, eles alternam entre plataformas e aplicativos para escapar à supervisão dos adultos, a fim de construir suas próprias biografias digitais sem censura.

\section{Considerações Finais}

Com base em evidências que indicam a probabilidade, mas não a certeza, de dano (STAKSRUD et al., 2013), a pesquisa parece ter sofrido uma reviravolta - começando a explorar uma abordagem diferente do risco; suavizando alguns pânicos digitais; assegurando que risco não significa, necessariamente, dano; e aceitando uma mediação que capacite as crianças para explorar oportunidades digitais, a fim de construir estratégias de enfrentamento e resiliência.

Este artigo baseia-se nas perspectivas de crianças portuguesas pré-adolescentes sobre como percebem a intervenção dos pais na sua atividade digital e reagem a ela. Com relação ao modo como os discursos da mídia moldam as percepções dos pais e a mediação do consumo de mídia digital pelos filhos, as vozes das crianças apontam que a mídia desempenha papel influente, o qual impacta na maneira como os pais formam percepções de risco e reagem, com esquemas de policiamento, às atividades digitais das crianças. Como, neste estudo, não tínhamos o objetivo de ouvir os pais quanto a essas questões, não podemos afirmar com certeza que a restrição e o policiamento sejam as únicas abordagens usadas por eles ou se, por sua vez, eles gerem e adaptam suas estratégias de acordo com as situações que possam surgir.

Sobre como respondem a abordagens de mediação parental mais intrusivas, apesar de tudo, as crianças defendem que os pais devem se preocupar com o que elas fazem on-line, mas também deixam claro que os pais devem respeitar seus direitos digitais à privacidade e à participação.

As respostas dos pais às possíveis ameaças digitais são construídas a partir de preocupações já ultrapassadas, que não atendem mais às vivências das crianças ou ao crescente cenário tecnológico 
em mudança no qual elas estão crescendo. Enquanto os pais se preocupam em proteger os filhos de possíveis danos, as narrativas das crianças mostram maior preocupação em se proteger do controle e da vigilância dos adultos, o que, em última instância, pode originar situações de maior complexidade, colocando em risco a própria proteção on-line das crianças e adensando o gap entre os dois grupos geracionais, como resultado de um desencontro de experiências e expectativas.

Ambos os grupos se beneficiariam de poder aprender em conjunto. Os adultos poderiam ganhar maior destreza tecnológica com as crianças e essas, beneficiar-se da sabedoria dos adultos a fim de ganhar competências para a segurança e o pensamento crítico, quais sejam: perceber a diferença entre as esferas pública e privada da vida pessoal; lidar com desafios que se colocam à sua segurança digital; e gerir relações interpessoais que facilmente atravessam do on-line para o offline (e vice-versa), prejudicando uma participação on-line saudável.

Por fim, as descobertas reforçam a necessidade de atualizar continuamente a pesquisa, para que possamos acompanhar os desenvolvimentos e as apropriações tecnológicas. A pesquisa sobre esse assunto tem forte impacto social, contribuindo com conhecimentos atualizados, materiais pedagógicos e exemplos de boas práticas que ajudem as famílias (além de educadores, pediatras, técnicos etc.) a proporcionar às suas crianças um apoio orientado e gradual, o qual promova a exploração máxima das oportunidades oferecidas pelo mundo digital (MASCHERONI et al., 2016).

\section{REFERÊNCIAS}

ANDERSON, A. Media and Risk. In: MYTHEN, G.; WALKLATE, S. (eds.). Beyond the risk society: Critical reflections on risk and human security. Maidenhead: Open University Press. 2006.

BECK, U. Risk society: Towards a new modernity. London: SAGE Publications Ltd., 1992.

BOND, E. Childhood, mobile technologies and everyday experiences - Changing technologies = changing childhoods? London: Palgrave MacMillan, 2014.

BOYD, D. It's complicated: The social lives of networked teens. New Haven/London: Yale University Press, 2014.

BOYD, D.; HARGITTAI, E. Connected and concerned: variation in parents' online safety concerns. Policy and Internet, Berkeley, v. 5, p. 245-269, 2013. https://doi.org/10.1002/1944-2866.POI332

BRAUN, V.; CLARKE, V. Using thematic analysis in psychology. Qualitative Research in Psychology, Philadelphia, v. 3, n. 2, p. 77-101, 2006.

CASTRO, T. “It's a complicated situation”. Harm in everyday experiences with technology. A qualitative study with school-aged children. 2015. $303 \mathrm{f}$. Tese (Doutorado em Tecnologia Educativa) Instituto de Educação, Universidade do Minho, Minho, 2015. Disponível em: http://hdl.handle. net/1822/40331. Acesso em: 14 set. 2020.

DUERAGER, A.; LIVINGSTONE, S. How can parents support children's internet safety? EU Kids Online, LSE Research Online, London, 2014. Disponível em: http://eprints.lse.ac.uk/42872/?from_ $\operatorname{ser} \mathrm{p}=1$. Acesso em: 14 set. 2020. 
FOUCAULT, M. Discipline and punish: The birth of the prison. New York: Vintage Books, 1979.

FRANKLIN, L.; CROMBY, J. “There might be 10 paedophiles sitting round in their front room": The $21^{\text {st }}$ Century Monster. Monsters and the Monstrous. Oxford: Inter-Disciplinary Net, 2009.

FUREDI, F. Culture of fear revisited: Risk-taking and the morality of low expectation. London: Continuum, 1997.

FUREDI, F. Paranoid parenting: Why ignoring the experts may be best for your child. London: Continuum, 2001.

GIDDENS, A. The consequences of modernity. Cambridge: Polity Press, 1990.

GILL, T. No fear: Growing up in a risk averse society. London: Calouste Gulbenkian Foundation, 2007.

GUBA, E.; LINCOLN, Y. Competing paradigms in qualitative research. In: DENZIN, N.; LINCOLN, Y. (eds.), The landscape of qualitative research: Theories and issues. Thousand Oaks: SAGE Publications Inc., 1998, p. 195-220.

HADDON, L. Mobile media and children. Mobile Media \& Communication, Los Angeles, v. 1, n. 1, p. 89-95, 2013. https://doi.org/10.1177/2050157912459504

HADDON, L. Children's critical evaluation of parental mediation. Cyberpsychology: Journal of Psychosocial Research on Cyberspace, Brno, v. 9, n. 1, 2015. https://doi.org/10.5817/CP2015-1-2

HELSPER, E. J. et al. Country classification: Opportunities, risks, harm and parental mediation. EU Kids Online, LSE Research Online, London, 2013. Disponível em: https:/www.lse.ac.uk/media@lse/ research/EUKidsOnline/EU\%20Kids\%20III/Classification/Home.aspx.

JAMES, A. Competition or integration? The next step in childhood studies? Childhood, London, v. 17, n. 4, p. 485-499, 2010. https://doi.org/10.1177/0907568209350783

JAMES, A.; JENKS, C. Public perceptions of childhood criminality. The British Journal of Sociology, London, v. 47, n. 2, p. 315-331, 1996. https://doi.org/10.2307/591729

LIM, S. S. Through the tablet glass: Mobile media, cloud computing and transcendent parenting. Journal of Children \& Media, London, v. 110, n. 1, p. 21-29,2016.https://doi.org/ 0.1080/17482798.2015.1121896

LIVINGSTONE, S.; BULGER, M. A global agenda for children's rights in the digital age Recommendations for developing UNICEF's Research Strategy. Florence: UNICEF Office of Research, 2013.

LIVINGSTONE, S. et al. Towards a better internet for children: Findings and recommendations from EU Kids Online to inform the CEO coalition. EU Kids Online, LSE Research Online, London, 2012. Disponível em: http://eprints.lse.ac.uk/44213 
LIVINGSTONE, S.; HELSPER, E. Parental mediation and children's Internet use. Journal of Broadcasting \& Electronic Media, Washington, v. 52, n. 4, p. 581-599, 2008. https://doi. org/10.1080/08838150802437396

LIVINGSTONE, S.; MASCHERONI, G.; STAKSRUD, E. Developing a framework for researching children's online risks and opportunities in Europe. EU Kids Online, LSE Research Online, London, 2015. Disponível em: http://eprints.lse.ac.uk/64470/

MAFFESOLI, M. The time of the tribes: The decline of individualism in mass society. London: SAGE Publications Ltd., 1996.

MASCHERONI, G. et al. Learning versus play or learning through play? How parents' imaginaries, discourses and practices around ICTs shape children's (digital) literacy practices. Media Education Studi, ricerche, buone pratiche, Trento, v. 7, n. 2, p. 261-280, 2016. https://doi.org/10.14605/MED721606

O’NEILL, B. Policy influences and country clusters: A comparative analysis of internet safety implementation. EU Kids Online, LSE Research Online, London, 2014.

PAIN, R. Paranoid parenting? Rematerializing risk and fear for children. Social \& Cultural Geography, Philadelphia, v. 7, n. 2, p. 221-243, 2006. https://doi.org/10.1080/14649360600600585

PASQUIER D. From parental control to peer pressure: Cultural transmission and conformism. In: LIVINGSTONE, S.; DROTNER, K. (eds.). International handbook of children, media and culture. London: SAGE Publications Ltd., 2008, p. 448-460.

PIPER, H.; POWELL, J.; SMITH, H. Parents, professionals, and paranoia: The touching of children in a culture of fear. Journal of Social Work, Los Angeles, v. 6, p. 151-167, 2006. https://doi. org/10.1177/1468017306066742

PONTE, C. et al. Crescendo entre ecrãs: Uso de meios eletrónicos por crianças (3-8 anos). Lisboa: Entidade Reguladora para a Comunicação Social, 2017. (Públicos e Consumos de Media.) Disponível em: http://www.erc.pt/documentos/Crescendoentreecras/files/downloads/crescendo-entre-ecras.pdf

PONTE, C.; SIMÕES, J. A. Asking parents about children's Internet use: Comparing Findings About Parental Mediation in Portugal and Other European Countries. EU Kids Online, LSE Research Online, London, 2009. Disponível em: www.fcsh.unl.pt/eukidsonline/docs/Asking\%20parents-FINAL\%20 Paper1_27-05-09.pdf

RIESSMAN, C. K. Thematic analysis. Narrative methods for the human sciences. Thousand Oaks: SAGE Publications Inc., 2008.

STAKSRUD, E.; ÓLAFSSON, K.; LIVINGSTONE, S. Does the use of social networking sites increase children's risk of harm? Computers in Human Behavior, New York, v. 29, n. 1, p. 40-50, 2013. https:// doi.org/10.1016/j.chb.2012.05.026 
STOKES, M. A. Stranger danger: Child protection and parental fears in the risk society. Amsterdam Social Science, Amsterdam, v. 1, n. 3, p. 6-24, 2009.

SUTTERBY, J. A. What kids don't get to do anymore and why. Childhood Education, Philadelphia, v. 85, n. 5, p. 289-292, 2009. https://doi.org/10.1080/00094056.2009.10521699

SUTTON, L. The state of play: Disadvantage, play and children's well-being. Social Policy and Society, London, v. 7, n. 4, p. 537-549, 2008. https://doi.org/10.1017/S147474640800448X

TYNES, B. M. Internet safety gone wild? Sacrificing the educational and psychosocial benefits of online social environments. Journal of Adolescent Research, Thousand Oaks, v. 22, n. 6, p. 575-584, 2007. https://doi.org/10.1177/0743558407303979

VANDONINCK, S.; D'HAENENS, L.; SMAHEL, D. Preventive measures - How youngesters avoid online risks. EU Kids Online, LSE Research Online, London, 2013. Disponível em: http://eprints. 1se.ac.uk/55797/

WONG, Y. C. Cyber-parenting: Internet benefits, risks and parenting issues. Journal of Technology in Human Services, New York, v. 28, n. 4, p. 252-273, 2010. https://doi.org/10.1080/15228835.2011 .562629

Recebido: 06 Jan. 2020

Aceito: 10 Jul. 2020

Editoras Associadas:

Adriana Laplane e Lucia Reily 